\title{
Comparative Analysis and Development Exploration of Threat Assessment Methods for Warship Air Defense
}

\author{
Pingbo $\mathrm{Yu}^{1}$, Gaofeng $\mathrm{Liu}^{1, \mathrm{a}}$ and Li Gong ${ }^{2}$ \\ ${ }^{1}$ College of Electronic Engineering, Naval Univ. of Engineering, 430033 Wuhan, China \\ ${ }^{2}$ Simulation Training Center, Naval Univ. of Engineering, 430033 Wuhan, China
}

\begin{abstract}
Warship's air defense target threat assessment is an uncertainty issue. This paper briefly states the basic process and types of threat assessment methods for warship air defense, using comparative research method, the mechanism and characteristics of the certainty/uncertainty assessment method for target threat assessment are compared. It also analyzes the application of fuzzy theory, neural network and genetic fuzzy tree in target threat assessment, which has certain reference value for the research of warship air defense target threat assessment.
\end{abstract}

\section{Introduction}

In today's sea battlefields, surface ships face threats from multi-domain such as airspace, maritime and underwater. Among them, airspace targets have strong advantages in speed, weapons, and tactics because of their unique attributes. Therefore, the importance of air defense operations for warships or warship formations is apparent [1-2]. In the face of the improvement of the effectiveness of air combat platforms, the speed of anti-ship weapons and the sharp increase of target information under saturated attack conditions, how to quickly and accurately assess airspace target threats is an important issue that needs to be solved urgently in warship air defense operations.

According to the characteristics of warship airspace target threats, the multi-objective evaluation method is commonly used in threat assessment. The multi-objective evaluation method has been studied and verified for a long time. So far, the most used methods are multiattribute evaluation method, technique for order preference by similarity to an ideal solution, the principal component analysis method, the Bayesian network method, the gray correlation analysis method, the cloud Model methods, etc.

This paper compares the target threat assessment methods from the perspective of information uncertainty in warship air defense target threat assessment, and forecasts the future development of target threat assessment.

\section{Airspace target threat assessment process and main methods}

\subsection{Basic process of target threat assessment}

\footnotetext{
a Corresponding author: gaofengliu_17@sina.com
}

In the course of combat, the target threat assessment is the premise of weapon allocation, providing decisionmaking basis for combat command [3-5]. When a warship faces a multi-target threat, it must give priority to hitting the target with the greatest threat. How to effectively judge the threat level of the target to the warship is a problem that needs to be solved in the threat assessment.

Airspace target threats in the sea battlefield environment can be divided into the following four categories [6]:

Table 1. The main threat of airspace targets in sea battlefield.

\begin{tabular}{|c|l|}
\hline Direct threats & $\begin{array}{l}\text { Anti-ship missiles, anti-radiation } \\
\text { missiles, guided bombs, etc. }\end{array}$ \\
\hline Indirect threats & $\begin{array}{l}\text { Intercepter fighters, bombers, attack } \\
\text { planes, helicopters, etc. }\end{array}$ \\
\hline Small threats & $\begin{array}{l}\text { Early warning aircraft, reconnaissance } \\
\text { aircraft, tankers, etc. }\end{array}$ \\
\hline Negligible threats & UFOs, flocks, false targets, etc. \\
\hline
\end{tabular}

The data sources of threat assessment are mainly shipborne sensors. The data information mainly includes the type of target, the speed of the target, the height of the target, the distance of the target, the chord angle of the target, the time of arrival, and the shortcut of the route [1$2][7-8]$ and so on. The judgment of the target threat level is based on the intention of the target, the number of targets, the degree of damage that may be caused to us, and so on. These evidences are descriptively summative and often difficult to obtain through direct observation, so they need to be judged by observable data. Through the calculation of the data fusion process and the evaluation method, the target threat level can be determined, which further provides a reliable guarantee for the automatic allocation of the weapon system. 


\subsection{The main methods of target threat assessment}

Existing work [9] divided the main methods of target assessment into mathematical analytical quantitative model methods and qualitative inference methods based on artificial intelligence theory. However, this classification is focused more on the methodological point of view and less on data information. Considering the realistic battlefield, the main methods of target threat assessment can be divided into certainty assessment methods and uncertainty assessment methods according to the degree of certainty of the target threat data, as shown in Figure 1.

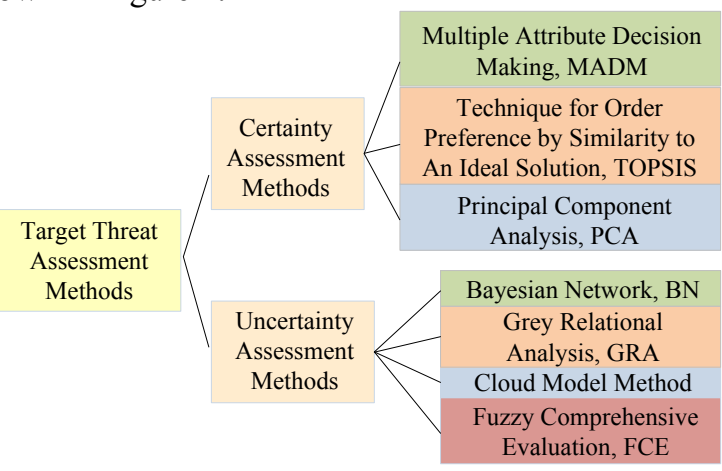

Figure 1. The Main Method of Target Threat Assessment

The certainty methods refer to the multi-objective threat assessment methods in the case where the battlefield situation is relatively clear and the indicators of the target threat are determined. The feature is that the operation is simple and easy to understand, and the evaluation result can provide a certain threat value. The uncertainty methods refer to the multi-objective threat assessment methods under the condition that the information is incomplete. The characteristic is that the threat value of the evaluation result is a probability solution or an interval solution, but because the requirements for the battlefield situation information are low, they can be used in special circumstances.

\section{Target threat assessment certainty methods comparison}

\subsection{Principle of typical methods}

In the case where the battlefield situation is already known, the certainty threat assessment methods can be used to make more accurate judgments on threats in the battlefield. In order to solve the problem that the dimensions of the original information are not unified, the data values obtained by real-time acquisition of various sensors (such as radar, sonar, etc.) on the warship should be pre-processed before the threat assessment, after the standardized processing, sort values according to mathematical rules and decision rules. Common methods for mathematical preprocessing include linear transformation, standard 0-1 transformation, vector normalization, and expert scoring method.
MADM is to use part of the attribute information of the target threats as the basis for evaluation, and weighting it by the index weight and mathematical method to evaluate it. The determination of index weights can be entropy method [10] and so on. The MADM methods generally appear in combination with other methods, such as TOPSIS method [8] and GRA method [11] as methods for determining the basis for evaluation.

TOPSIS is a method of sorting the proximity of each scheme to the ideal scheme (generally via selecting the Euclidean distance), and the value closest to the optimal solution and the farthest from the worst solution is the best choice. TOPSIS method is widely used, easy to calculate, and geometrically intuitive. The literature [7-8] uses TOPSIS method to calculate and rank the judgment indicators, and the calculation process is clear and easy to understand. The literature [12] establishes a threat assessment model based on the entropy weight-TOPSISgray correlation method. The entropy weight method is used to determine the objective weights of the evaluation index attribute, and the problem of relying on experience to give the attribute weights is solved. At the same time, according to the actual situation of the battlefield and the command demand, the gray correlation value can be further calculated based on the TOPSIS ranking. The combination of the two is judged to make the ranking result more convincing. Literature [13] introduces a theory of using punishment and incentive variable weights, which can compensate for the deviation caused by the constant weights. The weights of the evaluation criterions can be changed with the change of the state values of the evaluation criterions, thereby obtaining the decision matrix of the variable weights, and then sorting by TOPSIS method so that the result obtained is more sensible and reasonable.

PCA method is to select the main information in a large number of original battlefield situation information, eliminate the secondary information, extract the features by mathematical transformation, retain the original information as much as possible, and sort the newly obtained variables in descending order of variance. Literature [14] adopts a new dimensionless method, which makes the principal component retain more original information, but the calculation process is complicated. The literature [15] uses the kernel function to improve the feature extraction ability of PCA and reduce information loss. But the choice of the appropriate kernel function and nuclear parameters needs further determination.

\subsection{Characteristics of typical methods}

The certainty threat assessment methods can quickly analyze the threat with a simple and clear way when the situation between the enemy and us is clear, which can basically eliminate the need for the intervention of the commander in late period. With the development of technology and the equipment performance, the shipboard sensors' ability to perceive all kinds of information in the battlefield situation will increase, 
especially when we occupy the information initiative, they can play better roles.

The certainty target threat assessment methods can directly integrate the data obtained by the sensors, and the degree of automation is high, but there are still some shortcomings. The main shortcoming is that it is difficult to avoid the intervention of subjective factors, mainly in the process of selecting the basis of judgment, distinguishing the proportion of the judgment indicators from the overall judgment, and using the mathematical formula to project the initial data to the threat degree. The use of knowledge of expert system can only make the choice of methods as reasonable as possible, but still not fully objective. The TOPSIS ranking method also has a complicated process of finding a normative decision matrix. The calculation of the optimal solution and the worst solution sometimes fails to be solved. Both advantages and disadvantages of the PCA method are that the selection method of the original index is partial selection. When the original index is too much, the PCA method selects a small number of principal components for evaluation. On the one hand, the calculation is simple, and the burden of the evaluation system is reduced, but on the other hand, some indicators are also ignored, which may affect the overall judgment.

\section{Target threat assessment uncertainty methods comparison}

\subsection{Principle of typical methods}

Influenced by the anti-reconnaissance activities of the enemy on the battlefield, the concealment, randomness, complexity and other factors of the target threat cause information uncertainty, which has certain influence on the evaluation process. Therefore, it is also necessary to consider the threat assessment methods under uncertain conditions.

The Bayesian network is a directed acyclic graph. Each node has a conditional probability table indicating the probability of occurrence of subsequent node events. In [16], by constructing a threat assessment $\mathrm{BN}$ for target threat assessment of two targets, B-2 bomber and Boeing 747, the threat probability is obtained. Literature [17] established a dynamic BN threat assessment model, which can more accurately identify the threat level of the targets over time and improve the real-time performance of the BN threat assessment method.

The GRA is a method to measure the degree of correlation between two systems through the 'grey correlation degree', which is the closeness of the development trend between system factors. If the correlation value is close to 1 , it means that the changes of two systems are basically the same. The GRA method has a low demand for sample size, and is suitable for use in the case of incomplete information, with a small amount of calculation and a description of dynamic changes of the system. In [11], the positive and negative correlations in TOPSIS are used in the gray related closeness in the ranking, which weakens the influence of the indicators that have too much gray relation with the ideal value on the indicators with lower correlation. The contingency theory is introduced when considering the weights of each index, and the weights were updated with the actual state, which reduces the systematic error. In [18], the grey related degree between each attribute of the evaluation scheme and the reference scheme is calculated, and then the combined weights is calculated by the analytic hierarchy process and the entropy weight method, and the threat target rankings under different weights are calculated. Finally, compared with the expert system, the feasibility of this method is obtained.

The cloud model method is a method that uses a large number of cloud droplets to form a cloud image for possibility representation. It is geometrically intuitive. With each cloud drop represents uncertainty, the expected value of the cloud map is the quantitative value of the threat. Battlefield situation sometimes makes it difficult to obtain accurate data and information. In order to balance the randomness and ambiguity of real-time data on the battlefield, the literature [19] proposes a cloudbased target threat assessment method, which uses various cloud generators to form a processing system. The actual situation is abstracted into a cloud map for representation, and Bayesian feedback is used to correct the expert's assessment. In [20], the Bayesian network and cloud model are better combined with the cloud model for transformation from continuous to discrete, and then Bayesian network is used for reasoning, and finally the targets ordering is obtained. On the basis of the literature [20], the literature [21] further proposed the three-layer membership relationship of "cloud group, cloud family and cloud", and adopted the MIN-MAX cloud center of gravity inference algorithm to obtain the threat degree cloud drop through a large number of repeated inferences. Finally, the threat assessment is obtained through the cloud generator, which is very faulttolerant and robust.

\subsection{Characteristics of typical methods}

The uncertainty threat assessment methods are evaluation methods used when the battlefield situation is difficult to determine. When it is difficult to fully grasp the threat situation faced by the warship, the system needs to reason according to some data already grasped, therefore the judgment is given by means of interval, probability, and so on. This type of assessment methods can be used to conduct threat assessments on weapons with strong stealth and anti-reconnaissance capabilities.

The shortcoming of $\mathrm{BN}$ is that the process of determining the conditional probability value is complicated. It requires a large number of data samples for training. Some data sources rely on expert system knowledge, and subjective probability cannot be avoided. Besides, BN method requires independent evidence., otherwise they can't be used directly.

The GRA method needs to reflect the combination of qualitative and quantitative. Therefore, there is a certain error in the process of quantifying qualitative indicators in each judgment basis. Once the quantified value is changed, the final result will result in a gap. In addition, it 
is difficult to overcome the influence of subjectivity in the process of determining the weight matrix of the judgment basis.

The cloud model method requires a lot of repetitive calculations to obtain the cloud image, so the final result can judge the value from the graph. The purpose of the multiple calculation is to traverse all possible threat situations, and finally the cloud image expectation can be obtained. Therefore, the calculation process is complex and time consuming, and is not recommended to use when the battlefield situation is in emergency.

\section{Discussion on the development of target threat assessment method}

In the sea battlefield, the combatants of all parties must face the unfavorable situation away from replenishment. In particular, the combat forces such as surface ships must use limited resources to complete the attack on the target threats. With the change of war form, the uncertain factors faced by warships are more complicated than before. Compared with the order of threats of warship air defense targets, the sorting under uncertainty is a field that more scholars try to break through. Among them, the development of fuzzy theory and neural network in artificial intelligence provides a new idea for uncertainty threat assessment.

\subsection{Fuzzy theory and its application}

Fuzzy sets was proposed by Zadeh's in 1965, which is a set theory used to describe fuzzy objects. Fuzzy theory can be used to deal with fuzzy reasoning, uncertainty description, and paradoxical problems. In a fuzzy set, a membership function is used to describe the membership of an element $x$ belonging to a set A. Fuzzy theory is a method to study the problem of uncertainty.

In [22], the fuzzy set theory is established by using fuzzy set theory. The process of fuzzy reasoning involves membership function, logical operation and If-Then rules. By selecting threat information such as speed, altitude, distance, nearest point, maneuver, firepower, target support, and enemy and foe identification as input, and through the corresponding membership functions, the membership value falling in the interval $[0,1]$ is obtained, that is the threat degree. Literature [23] also proposed a method based on fuzzy reasoning (FRR) and fuzzy rough set to evaluate the airspace target threats.

Because fuzzy theory can better objectively represent the uncertainty of airspace targets and their operational intentions, it is widely used in decision-making of uncertainties. It can be predicted that the process from the uncertainty of airspace target threats to the certainty of decision-making will be one of the research trends in the future.

\subsection{Neural network and its application}

With the development of artificial intelligence, concepts and methods such as neural networks and deep learning have gradually been applied to various fields such as military, economic, and education. Neural network is a branch of machine learning. By simulating the learning process of the brain, constructing a perceptron, using input functions and transfer functions to give a network output. The neural network ranking method uses historical data to train the sorting process according to certain learning rules, and adjusts the network structure parameters, which can reduce the deviation caused by time and battlefield environment changes.

The use of neural network ordering methods requires three steps: determining the network structure, training sample input and parameter training. The characteristics and application directions of some common neural networks are shown in Table 2.

Table 2. Characteristics and applications of some common neural networks.

\begin{tabular}{|c|c|}
\hline $\begin{array}{c}\text { Common } \\
\text { neural } \\
\text { networks }\end{array}$ & Characteristics and applications \\
\hline $\begin{array}{c}\text { BP,Back } \\
\text { Propagation }\end{array}$ & $\begin{array}{l}\text { The input and output layers contain hidden } \\
\text { layers, which use error back propagation to } \\
\text { solve linear inseparable cases. }\end{array}$ \\
\hline $\begin{array}{l}\text { RBF, Radial- } \\
\text { Basis Function } \\
\text { Network }\end{array}$ & $\begin{array}{l}\text { The activation function is a feedforward } \\
\text { network of radial basis functions. } \\
\text { Applicable to function approximation. }\end{array}$ \\
\hline $\begin{array}{c}\text { MC, Maekov } \\
\text { Chain }\end{array}$ & $\begin{array}{l}\text { Each endpoint represents a possibility, } \\
\text { Applicable to finite state machines, } \\
\text { probability-based classification. }\end{array}$ \\
\hline $\begin{array}{c}\text { HN, Hopfield } \\
\text { Network }\end{array}$ & $\begin{array}{l}\text { A fully interconnected network, each neuron } \\
\text { is both an input and an output. Recursive } \\
\text { from the initial state to a steady state }\end{array}$ \\
\hline $\begin{array}{l}\text { BM, } \\
\text { Boltzmann } \\
\text { Machine }\end{array}$ & $\begin{array}{l}\text { A binary random machine consisting of } \\
\text { random neurons, and neurons and hidden } \\
\text { neurons are symmetrically connected, } \\
\text { seeking an optimal solution through } \\
\text { annealing algorithm }\end{array}$ \\
\hline $\begin{array}{c}\text { DBN, Deep } \\
\text { Belief Network }\end{array}$ & $\begin{array}{l}\text { Consisting of many Boltzmann machines, } \\
\text { generating data from samples that have } \\
\text { already learned. }\end{array}$ \\
\hline $\begin{array}{l}\text { DCN, Deep } \\
\text { Convolutional } \\
\text { Network }\end{array}$ & $\begin{array}{l}\text { With convolution unit and kernel, it can be } \\
\text { used to simplify input and reduce } \\
\text { unnecessary features, usually used for image } \\
\text { recognition }\end{array}$ \\
\hline $\begin{array}{l}\text { SVMs, } \\
\text { Support Vector } \\
\text { Machines }\end{array}$ & Used for linear two classification \\
\hline
\end{tabular}

The widely used neural networks are BP neural network and RB neural network. The BP algorithm uses a sigmoid logarithmic function, a hyperbolic tangent function, et cetera, as the activation function, and the RBF neural network uses a radial basis function (such as a Gaussian function) as the activation function.

In order to avoid the slow convergence and local minimization of BP neural network, the literature [24] uses the firefly optimization heuristic algorithm to obtain the initial weights and thresholds of BP neural network, and the result is compared with BP neural network, the error is reduced. Literature [25] uses PNN (Probabilistic Neural Network) to train data, and the final result is in line with the actual situation. In [26], BP neural network and Bayesian network are used to obtain both the dynamic and static weights of the judgment indicators 
respectively, and then the total threat degree is sorted by linear weighting.

In [27], the RBF network is used to train the parameters, and then the judgment indicators of different targets are tested. Compared with the expert method, the sorting obtained is more reasonable and effective. In [28], the improved RBF network is adopted, and the process of index processing is added, the corresponding indicators are selected according to the different combat style strategies. Although it has been greatly improved compared with the previous judgments, the selection of indicators is still not flexible enough, and this problem needs to be further resolved.

\subsection{Genetic fuzzy tree}

In June 2016, AlphaAI, an air combat artificial intelligence system developed by the University of Cincinnati and the US Air Force, defeated the US Air Force retired Colonel Gene Lee and attracted widespread attention [29]. AlphaAI uses the Learning Enhanced Tactical Handling Algorithm (LETHA) model. A visual LETHA genetic fuzzy tree includes the Route Planning Section, Weapon Systems Section, and Communication Constraints Section [30].

Each node of the genetic fuzzy tree is a fuzzy inference system (FIS). According to the evaluation function and stopping criterion selected by the fuzzy algorithm, the branch can be reduced. At the same time, the FIS is trained by the genetic algorithm, and the FIS is continuously optimized. The genetic fuzzy logic framework is shown in Figure 2. It is to use the genetic algorithm to complete the evolution process of the constructed fuzzy inference system, and finally obtain the fuzzy rules of the air combat strategy.

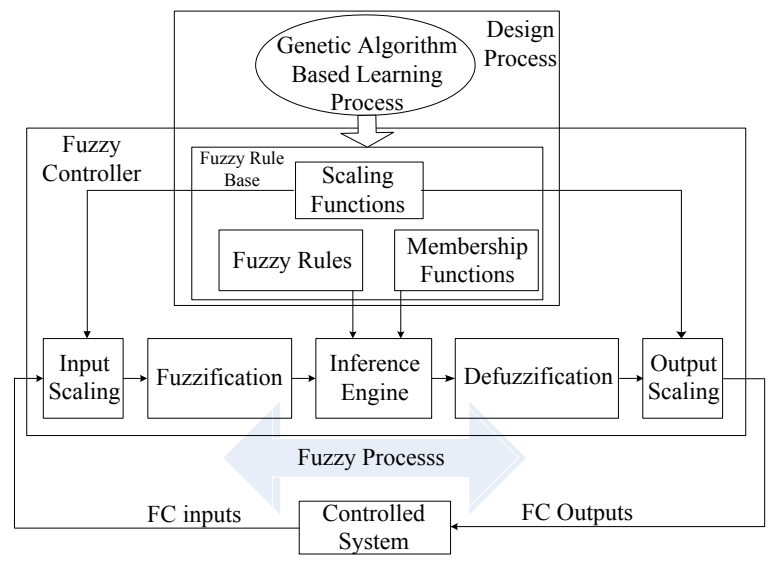

Figure 2. The Genetic Fuzzy Logic Framework

Traditional tools do not play their complexity and uncertainty well in the modeling process of complex systems, and fuzzy logic-based methods have been proven to model complex systems better. The method based on fuzzy logic can be used to represent the knowledge forms of different systems, and can also establish the relationship between system variables and interaction model between system [31].
Genetic algorithms are search algorithms that solve problems by imitating evolutionary processes in nature. Using the operations of selection, crossover and mutation, the process of genetic recombination of chromosomes is completed, gene pools are formed, the solutions that are not suitable for the problem are eliminated, and the genetically appropriate solutions are solved, so the method of solving the problem is completed in the end.

\section{Conclusion}

This paper briefly expounds the concept and classification of warship air defense target threats, and introduces MADM, TOPSIS, PCA, Bayesian network, GRA, and Cloud model method from two aspects: certainty and uncertainty method. From the perspective of future development, the fuzzy theory method, neural network method and genetic fuzzy tree method are prospected, which has certain guiding significance for the research on threat assessment methods of warship airspace targets.

The rapid development of computer technology has greatly improved the speed of data processing, and the parameter training process is more convenient and faster. Using an artificial intelligence approach which is closer to human thinking to assess the threat of a ship's airspace targets can help us build a more complete assessment model. The genetic fuzzy tree can complete the training of the target threat assessment rules. The threat value evaluation rules are input to the fuzzy controller through the control system to fuzzify, and the genetic algorithm is used to genetically evolve the evaluation rules until the set condition is reached, and then the fuzzy evaluation is performed, and the threat assessment rules at this time are output.

\section{References}

1. $\mathrm{R}$ Zhao, $\mathrm{Z}$ Liu, $\mathrm{S}$ Wang. Modern Defence Technology. 33, 15 (2005)

2. D Chen, Y Lei. Systems Engineering and Electronic. 27, 1597 (2005)

3. F Chang, X Zhang, Y Lin. Journal of Ordnance Equipment Engineering. 37, 160 (2016)

4. X Zhao, H Gu, Y Xiong. Fire Control \& Command Control. 8, 43 (2014)

5. P Zhang. Ship Electronic Engineering. 32, 31 (2012)

6. Y Song, Y Wang. Theory and Applications of Situation Assessment for Naval Air Defense (National Defense Industry Press, Beijing, 2014)

7. $\mathrm{Y}$ Lin, $\mathrm{G}$ Wang, Cong Wu, Ship Electronic Engineering. 36, 17 (2016)

8. W Wang, H Shi, L Wang, T Li. Modern Defence Technology. 42, 81 (2014)

9. H Huang. Ship Electronic Engineering. 34, 6 (2014)

10. K Pan, X Pan, X Guo. Computer \& Digital Engineering. 42, 803 (2014)

11. C Xia, D Zhou, Q Feng. Fire Control \& Command Control. 39, 53 (2014)

12. Y Zhang, W Ji, N Liu. Modern Defence Technology. 44, 73 (2016) 
13. $\mathrm{P} \mathrm{Du}, \mathrm{Y}$ Lu. Journal of Ordnance Engineering College. 29, 60 (2017)

14. S Zhu, D Zhou, Z Li. Computer Simulation. 27, 2 (2010)

15. D Li, L Jiang. Command Control \& Simulation. 38, 33 (2016)

16. Kumar S , Tripathi B K . Procedia Technology. 24, 1268 (2016)

17. H Bian, G Yang. Ordnance Industry Automation. 34, $14(2015)$

18. Y Zhao, W Li, B Liu, C Cui. Journal of Detection \& Control. 36, 80 (2014)

19. S Ma, L Han, G Gong, X Song. Journal of Beijing University of Aeronautics and Astronautics. 36, 150 (2010)

20. Y Zhang, B Li, J Cui. Computer Science. 40, 127 (2013)

21. Y Zhang, B Li. Journal of System Simulation. 26, 411 (2014)
22. Ehsan Azimirad, Javad Haddadnia. International Journal of Advances in Intelligent Informatics. 1, $57 .(2015)$

23. Y Lu, Y Wang, Y Lei, Y Wang. 2015 27th Chinese Control and Decision Conference (Qingdao, 2015)

24. X Wang, Q Zhu, A Tan, Y Zhang. Command Control \& Simulation. 36, 42 (2014)

25. K Dong, G Yang, Z Wang. Modern Defence Technology. 39, 71 (2011)

26. C Han, H Yang, C Tu. Computer Simulation. 34, 54 (2017)

27. F Zhang, D You, M Jiang. Shipboard Electronic Countermeasure. 33, 85 (2010)

28. K Xu, D Kong, J Chen. CIAC. (Fuzhou, 2015)

29. G Zhou, F Zhou. The Sixth China Conference on Command and Control. (Beijing, 2018)

30. Ernest N, Cohen K. Defense Management. 6, 139 (2015)

31. Herrera F, Magdalena L. World Scientific. 46488 (2007) 